The University of Southern Mississippi

The Aquila Digital Community

Faculty Publications

7-8-1996

\title{
Sol-Gel Phase Transitions in Thermoreversible Gels: Onset of Gelation and Melting
}

Y. Liu

University of Southern Mississippi

R.B. Pandey

University of Southern Mississippi

Follow this and additional works at: https://aquila.usm.edu/fac_pubs

Part of the Science and Mathematics Education Commons

\section{Recommended Citation}

Liu, Y., Pandey, R. (1996). Sol-Gel Phase Transitions in Thermoreversible Gels: Onset of Gelation and Melting. Journal of Chemical Physics, 105(2), 825-836.

Available at: https://aquila.usm.edu/fac_pubs/5611

This Article is brought to you for free and open access by The Aquila Digital Community. It has been accepted for inclusion in Faculty Publications by an authorized administrator of The Aquila Digital Community. For more information, please contact Joshua.Cromwell@usm.edu. 


\title{
Sol-gel phase transitions in thermoreversible gels: Onset of gelation and melting
}

\author{
Y. Liu \\ Program in Scientific Computing, University of Southern Mississippi, Hattiesburg, Mississippi 39406-5046 \\ R. B. Pandey \\ Program in Scientific Computing and Department of Physics and Astronomy, \\ University of Southern Mississippi, Hattiesburg, Mississippi 39406-5046 \\ and Computational Science Programme, The National University of Singapore, \\ Lower Kent Ridge Road, Singapore 119260
}

(Received 3 January 1996; accepted 1 April 1996)

\begin{abstract}
A Monte Carlo simulation model is proposed to study the phase transition and the structural evolution of thermoreversible gels with the coexistence of phase separation and gelation processes. Our model includes the mobility of all the species and the reversibility of bonds of the clusters formed due to cross-linking reactions. These features provide a more realistic description of a polymer-solvent system. We attempt to elucidate the effects of interactions, solvents, polymer fraction, etc. on the phase behaviors of the thermoreversible gel. Sol-to-gel transition is studied in detail as a function of temperature, and the related critical exponents are evaluated. Two different energy parameters are used to describe the gelation and the melting processes. The collective structure factors are calculated, and their dynamic behaviors are analyzed. The competing effects of the phase separation and the network formation on the structural evolutions of the gel are discussed. (C) 1996 American Institute of Physics. [S0021-9606(96)50226-6]
\end{abstract}

\section{INTRODUCTION}

A thermoreversible gel is a three-dimensional network of polymer chains cross-linked by physical junctions through various mechanisms. The energy associated with the physical junctions is of the order of thermal energy $k_{B} T$ so that the system can readily reach thermal equilibrium. Flory ${ }^{1}$ classified the thermoreversible gels as polymer networks formed through physical aggregations. The junction points consist of physical bonds. Eldridge and Ferry ${ }^{2}$ considered junctions as hydrogen-bonding type associations of polymer chains. Many studies have shown that the processes by which junction points are created can be complex. In gelatin gel, junction points are triple helices. The molecular forces that make these helices aggregate are generally believed to be secondary forces such as hydrogen bonds. ${ }^{3}$ In the case of the crystalline polymer, crystallites or fringed micelles act as junction points. The gelation of crystalline polymers is more complex than that of amorphous polymers. Although the type of associations which results in the gel network is varied with systems, a mechanism of physical cross-linking is a necessary requirement for the formation of thermoreversible gels.

The thermoreversible gelation of polymer solutions is essentially an equilibrium phenomenon. Normally, gelation occurs with decreasing temperatures, while a gel melts at elevated temperatures. Thermoreversible gels exhibit a variety of interesting phases, and may undergo a phase transition from a sol state to gel state (gelation) on cooling and a phase transition from a gel state to a sol state (gel-melting) on heating. Many polymer solutions undergo liquid-liquid phase separation on cooling. It is believed that this results from a competition between the temperature and the polymer-polymer and the polymer-solvent interactions such as hydrogen-bonding and hydrophobic interactions. Figure 1 shows a phase diagram observed in thermoreversible gels. This diagram displays the general qualitative features of phase transition for a gelating system in a semidilute solution with a good solvent and was first studied by de Gennes. ${ }^{3}$ Tan et al. ${ }^{4}$ verified the diagram experimentally for a noncrystallizable polymer, atactic polystyrene (aPS). Kawanishi et al. ${ }^{5}$ gave a general description of the phase behaviors of thermoreversible gels.

The behaviors of thermoreversible gelation can be examined from two points of view: thermodynamic and structural behaviors. Extensive experiments have been carried out to investigate the thermal properties of thermoreversible gels by laser-light scattering and fluorescence spectroscopy. The effects of polymer weight, concentration, etc. on the gelation temperature and gel melting temperature have been investigated intensively. ${ }^{6-23}$ The gel structure created by phase separation has been observed in many polymer systems. ${ }^{24-37}$ The dynamic development of phase separation and the final structure of gels were found to depend strongly on the temperature and the cross-linking rate. The interplay between the phase separation process and the gelation process leads to interesting structural morphology in thermoreversible gels.

Several theoretical attempts have been made to describe the kinetics of the thermoreversible gelation with phase separation. Using a lattice theory, a multicriticality model was introduced by Tanaka et al. ${ }^{38}$ The model is based on the Flory-Huggins solution theory and statistics of chain reaction and has the characteristics of a mean-field theory, which neglects the spatial correlation in a real system, where large spatial fluctuations in the cross-link density may determine 


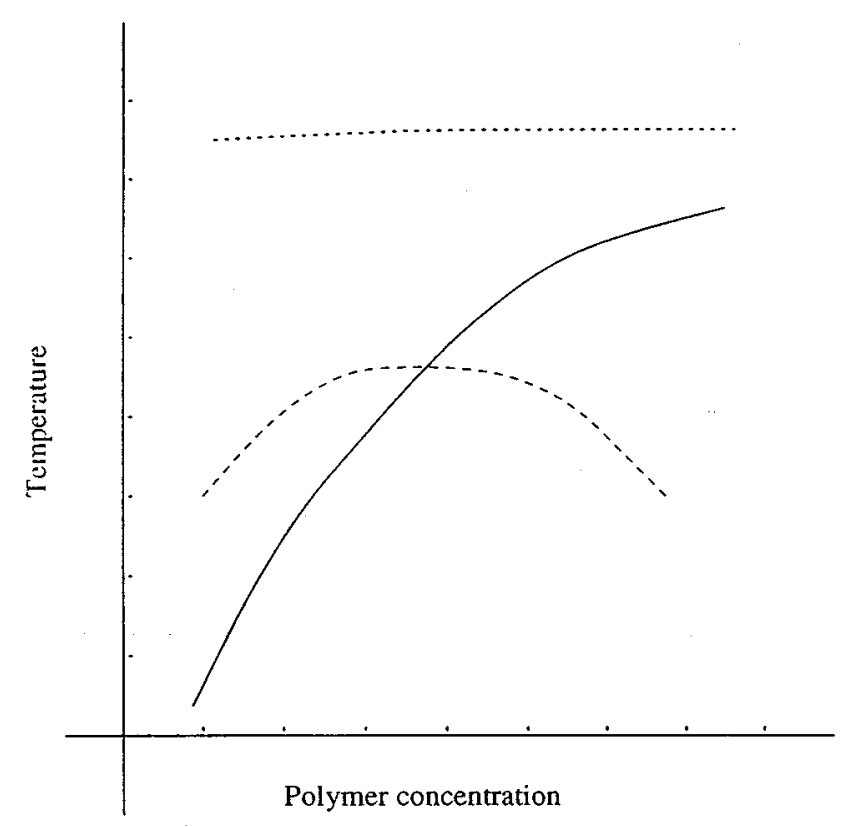

FIG. 1. A phase diagram observed in thermoreversible gels (solid-line: gelation curve; dash-line: spinodal curve; dot-line: gel-melting curve).

the behavior of the gelation process. A model for reversible gelation based on the correlated percolation was proposed by Coniglio et al. ${ }^{39}$ As an extension of this model, two disparate energy scales were used to describe the phase separation in the gelation processes. ${ }^{40}$ The Ginzburg-Landau-type equation was also used to describe the kinetics of gelation along with the phase separation. ${ }^{41}$ These analytical theories have a problem in mapping the parameters of the models into the real systems. Because of the complex nature of the thermoreversible gelation process, it is difficult to find a simple relation between the factors that affect the structure of gel networks experimentally. In this sense, a computer simulation has the advantage of studying the system under well controlled conditions. Computer simulations based on the Isingcorrelated site-bond percolation model were used to study the effect of temperature on the gelation. ${ }^{42,43}$ In these models, the monomers are distributed in thermoequilibrium at the temperature $T$. The probability for two monomers at nearestneighbor distance to form a bond depends on two variables: temperature and concentration. Several computer simulation models have been proposed to study the phase separation in gelation processes in a variety of polymer systems. ${ }^{44-46}$ In some of these computer simulation models, the mobility of polymers was not considered, while some models were concerned only with the homogeneous network and neglected the fluctuation in the cross-link density. The inhomogeneities of gel networks were observed and studied in the kinetic gelation model ${ }^{47,48}$ particularly in an irreversible growth where the inhomogeneities result from an unequal reactivity of the system, where bonds are formed by the random motion of radicals. No temperature was involved.

In this paper, we study the thermal behaviors and the structural properties of thermoreversible gels using computer simulations. We attempt to incorporate the main idea of the thermodynamic theories of thermoreversible gelations. We consider both the mobility of polymers and the reversibility of bonds in gelation processes. In our model, the gelation and phase separation are due to temperature quenching. This is very close to a real gelling system. We deal with the connectivity of gel networks in this paper. The chemical details in the formation of junctions are neglected. In the following Sec. II the method is described in detail for two systems: (a) a binary mixture of monomers and solvent, and (b) a mixture of polymer chains, solvent, and empty sites. The results and discussion of these simulations are presented in Sec. III, with conclusions in Sec. IV.

\section{METHODS}

We consider a simple cubic lattice of size $L \times L \times L$ with a periodic boundary condition. A fraction $C_{p}$ of the lattice sites is randomly occupied by monomers (statistical units) in system (a). Each monomer carries a number $(f)$ of reactive groups (functionality) capable of forming bonds pairwise. These units can be pre-bonded to become polymer chains in system (b). In this case, the number of the chain is equal to $C_{p} \times L^{3} / n$, where $n$ is the length of the chain. Then a fraction $C_{0}$ of the lattice sites is occupied by solvent particles. The fraction $1-C_{p}-C_{0}$ of the lattice sites is left empty. A lattice site cannot be occupied by more than one unit at a time. A polymer unit can react with the units in other polymers and form a bond (cross-link) between them, but it cannot react with any unit of its own chain.

We consider an attraction between non-bonded neighboring polymer units. This attractive potential is expressed through a reduced energy $\epsilon_{p p} / k_{B} T$. Polymer-solvent interactions are repulsive, described by $\epsilon_{s p} / k_{B} T$. The solventsolvent attractions are described by $\epsilon_{s s} / k_{B} T$. We only consider the surface interactions between polymer clusters (i.e., microgel particles) formed by aggregations and the neighboring monomers and/or solvent particles.

\section{A. Mobility of species}

The concentration of the vacancies is usually greater than $50 \%$ in this study. Thus the species in the system can have central-mass diffusion (translational diffusion). Monomers and microgel particles (a finite cluster resulting from the reaction) can move one lattice unit to a neighboring vacant space in a randomly selected direction (one of six simple cubic directions) in one attempt. During the move, all bonds are preserved and the conformation of the polymer remains unchanged. The diffusion coefficient $D_{n}$ for a free cluster with $n$ monomers is given by $D_{n} \sim 1 / n$, i.e., the hopping rate of a cluster is inversely proportional to its mass.

The motion of a polymer is implemented by selecting a unit randomly and performing a simple bond fluctuation or reptation depending on the location of the node/unit (i.e., interior node or the ends, respectively) in a chain. An interior monomer of a bonded unit can diffuse in a selected direction as long as the bond length $\left(l_{b}\right)$ is preserved within a certain limit: $1 \leqslant l_{b} \leqslant \sqrt{3}$. An unreacted chain can reptate if the ran- 


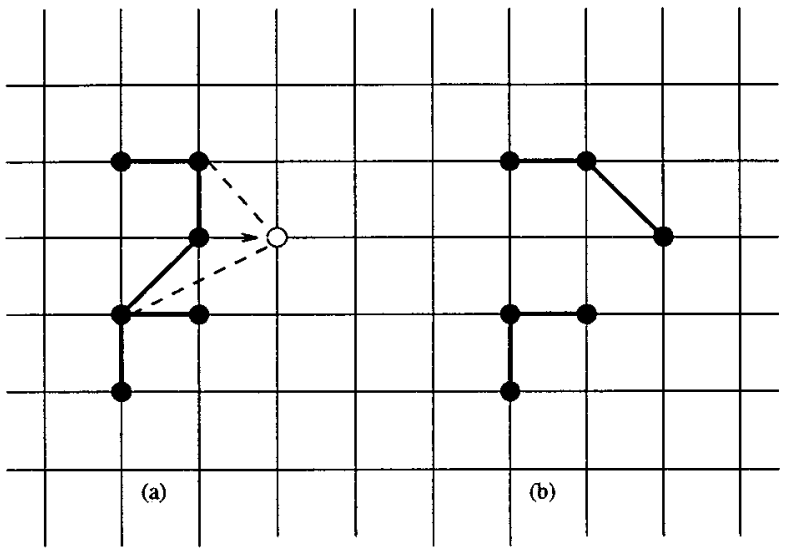

FIG. 2. A example of the breaking of bonds due to the displacement moves of monomers. (a) Before the breaking; (b) after the breaking.

domly selected unit is at one end of the chain, and the selected direction of the move is the same as the bond-pointed direction. A solvent particle can move to its randomly selected neighboring empty site in the lattice. Further, if a solvent particle is on the way of moving a cluster, then the solvent particle is moved in the direction of the cluster's motion to make room for the incoming cluster-a collision effect.

The energy associated with monomer/polymer units will affect the motion of the molecules and thus the formation of a bond (cross-link). The moving probability depends only on the energy change due to move, via $e^{-J_{m} \Delta E / T}$, where $\Delta E$ is the energy difference between the new and the old configurations and $J_{m}$ is the interaction strength. The acceptance and rejection of the motion of monomers and microgels is decided by the Metropolis algorithm. In this method the states generated by each move are highly correlated with the previous states. The mobility of the monomers and the solvent particles is controlled by their interaction energy, and thus, by the thermodynamics through the transition probability $W_{m}=e^{-J_{m} \Delta E / T}$. Since the collision probability of reactive groups is controlled by the cluster mobility, polymer units or microgel particles tend to move close to each other and form bonds at lower temperatures.

\section{B. Reversibility of cross-links}

In a thermoreversible gelation, a cross-link (junction point) can be formed and destroyed by thermal motions. The binding energy connecting a pair of the polymer segments is expected to be of the order of thermal energy. In this model, a cross-link can be broken due to displacement of a polymer unit at the cross-link. When a polymer unit at a junction point attempts to move in a randomly-chosen direction and the move is accepted energetically, then the cross-link will be broken up if the bond length is stretched beyond $\sqrt{3}$. A possible breakup of a cross-link in a cluster is illustrated in Figure 2. In addition to this criterion of bond stretching beyond $\sqrt{3}$, we implement a thermodynamic criterion to break a bond. It is assumed that the stability of physical cross-links between polymer segments with respect to thermal motion is primarily determined by the ratio of the energy $\left(J_{r} \Delta E\right)$ required to break the cross-link and the thermal energy $k_{B} T$, i.e., by the transition probability $W_{r}=e^{-J_{r} \Delta E / T}$, where $J_{r}$ is a measure of the magnitude of the breakup energy related to the potential between polymers or solvents. Because of an attractive potential between the polymer units, a unit has to overcome potential barriers to move away from the neighboring units. To consider the bond strength, we use an additional probability of thermal reversibility $\left(p_{r}\right), p_{r}=e^{-c / k_{B} T}$, where $c$ is a constant which is a measure of the physical bond strength. So the actual transition probability for breaking a bond is $W_{r}^{\prime}=W_{r} \times p_{r}$, where $W_{r}$ is the thermodynamic transition probability as in the Metropolis method. In any case we note that the reversibility will increase as the temperature is elevated.

\section{Formation of network}

We assume that each unit in the system has equal reactivity. The bond is formed between reactive functional groups of a unit by a method similar to a chemical controlled kinetic reaction. A bifunctional monomer can be connected to its two neighboring monomers at the most by single bonds and to one neighboring monomer at the most by a double bond. Similarly, a tetrafunctional monomer can be connected to its four neighboring monomers at the most by a single bond, to one monomer at the most by four bonds, or to its neighboring monomers by various bonding with multiplicity between one and four.

The system starts with randomly distributed polymer chains or monomers. To evolve the system, we select each unit (bonded or unbonded) randomly and attempt to move it by a lattice unit distance in a randomly chosen direction. This move may cause breakup of a cross-link already formed. We also select a cluster randomly and attempt to move it with its hopping probability in a randomly chosen direction. The acceptance of the move is governed energetically as explained above. After hopping, each unit attempts to react with one of its randomly selected neighbors. If the neighboring site is occupied by a unit and both units have at least one unsaturated bond (i.e., unreacted functional unit), then a bond is formed between the two monomers with a certain bonding probability $p_{b}$. An attempt to form a bond fails with probability $\left(1-p_{b}\right)$ or if the randomly selected neighboring site is not occupied by a polymer unit or if either of the units is saturated. A unit becomes saturated as soon as it bonds with all of its functional groups. This process of hopping each particle by one step and attempting to form bonds is repeated again and again until most of our attempts to form bonds fail, i.e., when the reaction is nearly complete. The time unit is measured by the Monte Carlo steps (MCS). The MCS is defined as attempts to move all the monomers and clusters and to let them react with their nearest neighbors. One MCS can be divided into $m$ intervals, in which 
only $1 / m$ of the total species can be randomly selected to diffuse and react.

In the course of the kinetic growth, the concentration of the reacted monomers increases. So does the extent of reaction or the extent of cross-linking $p$ which is defined as the ratio of the number of cross-links and the maximal possible number of cross-links. At the sol-gel transition threshold, $p_{c}=p\left(t_{c}\right), p_{c}$ and $t_{c}$ are the critical bond concentration and critical time, respectively.

\section{Model properties}

The process of gelation is related to connectivity properties of the system. When a cluster spans the lattice, the system undergoes sol-to-gel transition. This geometrical transition can be studied as a function of time $(t)$, the extent of reaction [fraction of bonds $(p)$ ], and the temperature $(T)$. A thermal transition is induced when temperature is varied. We choose the gel fraction as the order parameter of the system, which is defined as

$$
G=\frac{\phi_{G}}{\phi_{S}+\phi_{G}}=\langle|G|\rangle=\frac{n_{G}}{N},
$$

where $\phi_{G}$ and $\phi_{S}$ are the fractions of the two components in the system: gel cluster and finite clusters in sol, i.e., $\phi_{G}=n_{G} /\left(n_{S}+n_{G}\right)$ and $\phi_{S}=n_{S} /\left(n_{S}+n_{G}\right)$, where $n_{G}$ and $n_{S}$ are the number of monomers in gel and sol, respectively, and $n_{G}+n_{S}=N$ is the total number of polymer units. Since the network is flexible via bond fluctuation and is in constant thermal motion, aggregations of polymer are constantly forming and disintegrating. In the critical regime (around the critical temperature), the average total number of bonds may not change, while $n_{G}$ fluctuates since the bonds participating in the gel network are in equilibrium. After the relaxation time $t_{\tau}$, a balance will be established between the bonding and break-up processes, leading to an equilibrium state in which the order parameter is independent of time $\left(t \gg t_{\tau}\right)$.

Besides the order parameter $G$, we also keep track of the following quantities: (1) Mean gel size (Weight-average degree polymerization) $M_{W}$ which is the ratio of the second moment to the first moment of the mass distribution, i.e.,

$$
M_{W}=\frac{\sum_{s} n_{s} s^{2}}{\sum_{s} n_{s} s}
$$

where $n_{s}$ is the number of clusters containing $s$ monomers. (2) Correlation length ( $\xi$ ) (or $z$-average of the radius), which is the measure of the spatial extension of the connectivity, is given by

$$
\xi^{2}=\frac{\sum s^{2} n_{s} R_{s}^{2}}{\sum s^{2} n_{s}}
$$

where $R_{s}$ is the radius of gyration defined as

$$
R_{s}^{2}=\frac{1}{s}\left\langle\sum_{i=1}^{s}\left(\mathbf{r}_{i}-\mathbf{r}_{0}\right)^{2}\right\rangle,
$$

with $\mathbf{r}_{0}=\sum_{i=1}^{s} \mathbf{r}_{i} / s$ and $\mathbf{r}_{i}$ denotes the position of the $i$ th connected site.
The structure factors are widely used to investigate the structural development of polymer solutions and gel networks. The structure factor of an $L^{3}$ lattice is the Fourier transformation of the spatial correlation function which measures the difference between the local concentrations of two components in the system: $\operatorname{polymer}\left(\phi_{p}\right)$ and solvent $\left(\phi_{s}\right)^{49-52}$

$$
S(\vec{q}, t)=\frac{1}{L^{3}}\left\langle\left\{\sum_{\vec{r}} e^{i \vec{q} \cdot \vec{r}}\left[\left(\phi_{p}-\phi_{s}\right)-<\phi_{p}-\phi_{s}>\right]\right\}^{2}\right),
$$

where $\vec{r}$ runs over all lattice sites and

$$
\vec{q}=\left(\frac{2 \pi}{L}\right) \vec{\mu}=\frac{2 \pi}{L}\left(u_{x}, u_{y}, u_{z}\right), u_{x}, u_{y}, u_{z}=0 \cdots L .
$$

A local concentration variable $\phi_{p}^{j}$ is equal to 1 if the lattice site $j$ is occupied by a polymer unit and otherwise zero, and $\phi_{s}^{j}$ is equal to 1 if it is taken by a solvent particle and otherwise zero. In order to investigate the time evolution of the structure, we compute the collective structure factor of the system, which is the spherical average of $S(\vec{q}, t)$ in a spherical shell of radius $q$ with 1 lattice unit.

$$
S(q, t)=\sum_{q} S(\vec{q}, t) / \sum_{q} 1,
$$

for $q=\frac{2 \pi}{L} n$. The sum $\Sigma_{q}$ goes over all values of $\vec{q}$ such that

$$
\frac{2 \pi}{L} n \leqslant|\vec{q}|<\frac{2 \pi}{L}(n+1) .
$$

We use $n=1,2, \ldots, 10$ in our calculations. We are also interested in the structure factor $S_{1}(q, t)$ for the solvent which may describe a possible solvent-vacancy phase separation

$$
S_{1}(\vec{q}, t)=\frac{1}{L^{3}}\left(\left\{\sum_{r} e^{i \vec{q} \cdot \vec{r}}\left[\phi_{s}-<\phi_{s}>\right]\right\}^{2}\right\rangle .
$$

\section{RESULTS AND DISCUSSIONS}

\section{A. Thermal transitions in a monomer-solvent mixture}

In this section, we shall investigate the critical properties of thermoreversible gels in a binary mixture of monomers and solvents. We consider two kinds of monomers, one with functionality $f=2$ and another with $f=4$. A fraction $C_{2}$ of the lattice sites is randomly occupied by bifunctional monomers and a fraction $C_{4}$, by tetrafunctional units, leaving the remaining fraction $1-C_{2}-C_{4}$ of lattice sites empty. The empty sites can be considered to be occupied by solvents. We shall choose the fraction of the two monomers, $C_{2}=0.1$ and $C_{4}=0.3$ throughout this section. Polymer-solvent interactions are not explicitly included here. We choose the energy parameter $J_{m}=10$, which will affect the mobility of the components in the system, and the breakup energy parameter $J_{r}=10$. The bonding probability $\left(p_{b}\right)$ is set to be 0.8 .

We know that most thermoreversible gelations occur on the cooling the system, i.e., on reducing the temperature. To investigate this gelation process, we start with the infinite 


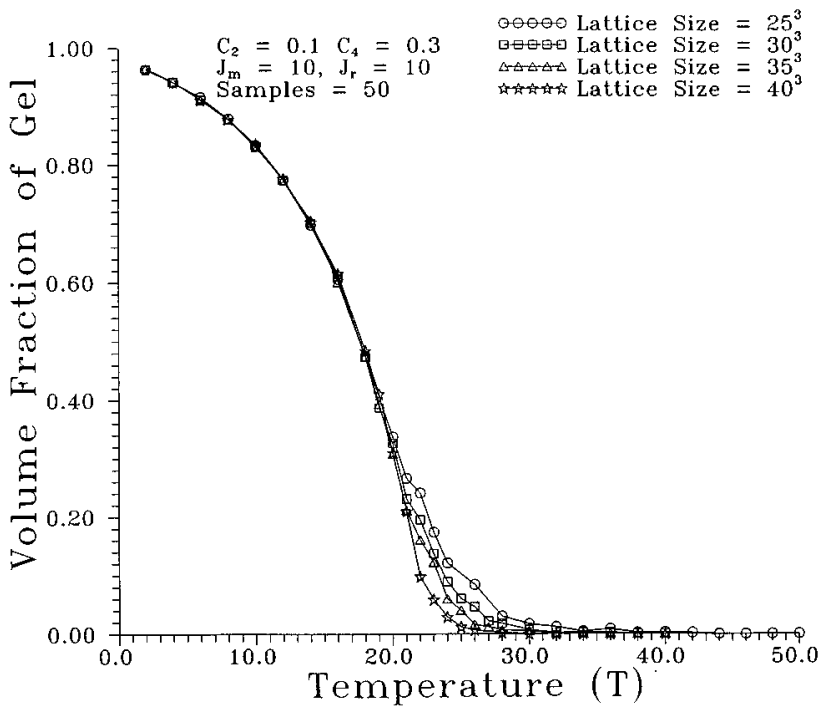

FIG. 3. Gel volume fraction $(G)$ vs temperature $(T)$ on the lattice of various sizes: $25^{3}, 30^{3}, 35^{3}, 40^{3}$ with $J_{m}=10, J_{r}=10$.

temperature (i.e., with the polymer units distributed randomly in the lattice) where no cluster has been formed (in the sol phase). Then we let the system evolve at a certain temperature $T$ with the two energy parameters: $J_{r}$ and $J_{m}$. After a relaxation time, the system will equilibrate. Then we propagate the system by $20 \mathrm{MCS}$, during which the statistical averages of the physical quantities were determined. Repeating this procedure at various temperatures, we obtain the relation between the physical properties of the system and temperatures.

In order to test the finite size effect, we perform simulations on lattices of various sizes. Figure 3 shows the variations of gel fraction vs temperature with various lattice sizes. The order parameter $G$ would be zero for all temperatures $T>T_{c}$. The gel fraction $G$ has a power law with a critical exponent $\beta$ near the gelation threshold

$$
G \sim\left(1-T / T_{c}\right)^{\beta} .
$$

We observe a finite size effect on the critical temperature. The variations of the mean gel size with various lattice sizes are shown in Fig. 4. This quantity diverges at $T_{c}$ with a critical exponent $\gamma$

$$
M_{W} \sim\left(1-T_{c} / T\right)^{-\gamma} .
$$

The divergence of the correlation length at $T_{c}$ is described by an exponent $\nu$,

$$
\xi \sim\left(1-T_{c} / T\right)^{-\nu}
$$

It is generally believed that phase transitions have certain universal properties such that the critical exponents are independent of the microscopic details of the systems ${ }^{42}$ in the critical region. We have attempted to estimate the critical exponents using the finite-size scaling analysis method. ${ }^{53,54}$ The gel fraction $G$ and mean gel size $M_{W}$ can be expressed in terms of scaling function $\widetilde{G}$ and $\widetilde{M}_{W}$

$$
G L^{\beta / \nu}=\widetilde{G}\left[\left(1-T / T_{c}\right) L^{1 / \nu}\right],
$$

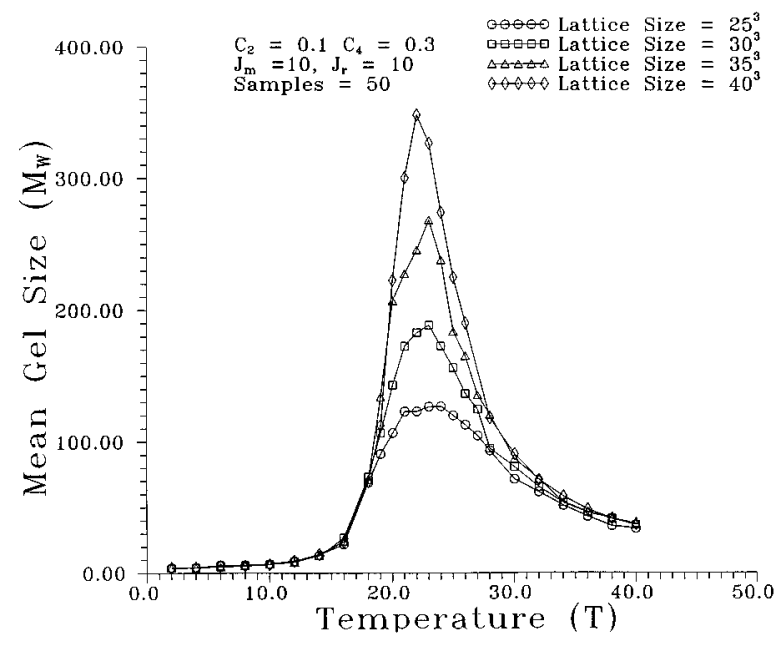

FIG. 4. Mean gel size $\left(M_{W}\right)$ vs temperature $(T)$ on the lattice of various sizes: $25^{3}, 30^{3}, 35^{3}, 40^{3}$ with $J_{m}=10, J_{r}=10$.

$$
M_{W} L^{\gamma / \nu}=\widetilde{M}_{W}\left[\left(1-T_{c} / T\right) L^{1 / \nu}\right],
$$

when $T \rightarrow T_{c}$ and $L \rightarrow \infty$. Figures 5 and 6 are the finite-size scaling plot of $G$ and $M_{W}$, which shows that the family of curves for $G$ and $M_{W}$ collapse on corresponding curves for functions $\widetilde{G}$ and $\widetilde{M}_{W}$ with the choice of $T_{c}=23, \beta=0.58$, $\gamma=1.42$ and $\nu=0.73$. These values are close to those in the percolation model.

The gelation temperature depends upon the concentration of the polymer units in the system. The effects of concentration of monomers on the phase transitions are shown in Fig. 7 which plots the gel fraction vs temperature for concentrations of monomers $C=0.3,0.4$, and 0.45 $\left(C_{2}: C_{4}=1: 3\right.$ and $\left.C_{2}+C_{4}=C\right)$. It follows from the data that the gelation temperature increases as the concentration of monomers increases. The gelation temperature versus the monomer concentration is shown in the phase diagram (see Fig. 8).

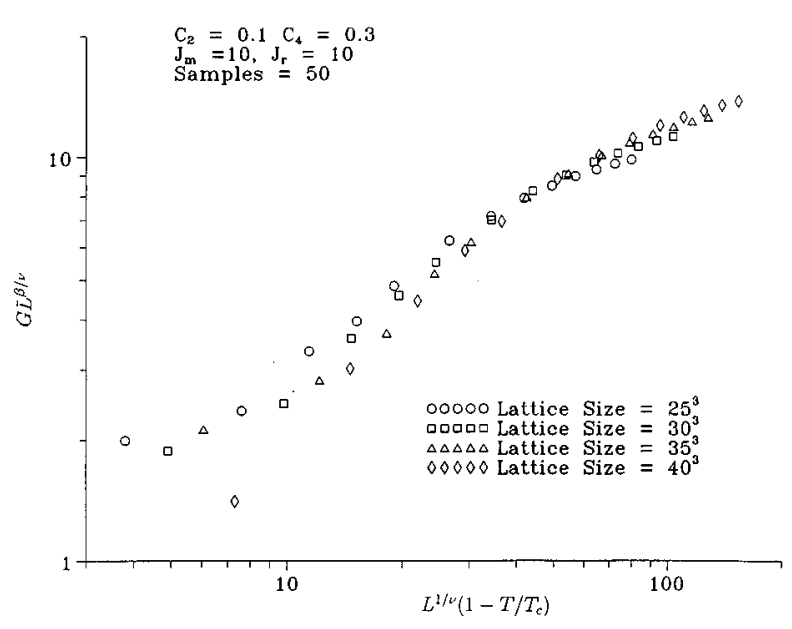

FIG. 5. Log-log plot of $G L^{\beta / \nu}$ vs $\left|1-T / T_{c}\right| L^{1 / \nu}$ with $J_{m}=10, J_{r}=10$. Assuming that $\beta=0.58, \nu=0.73$. 


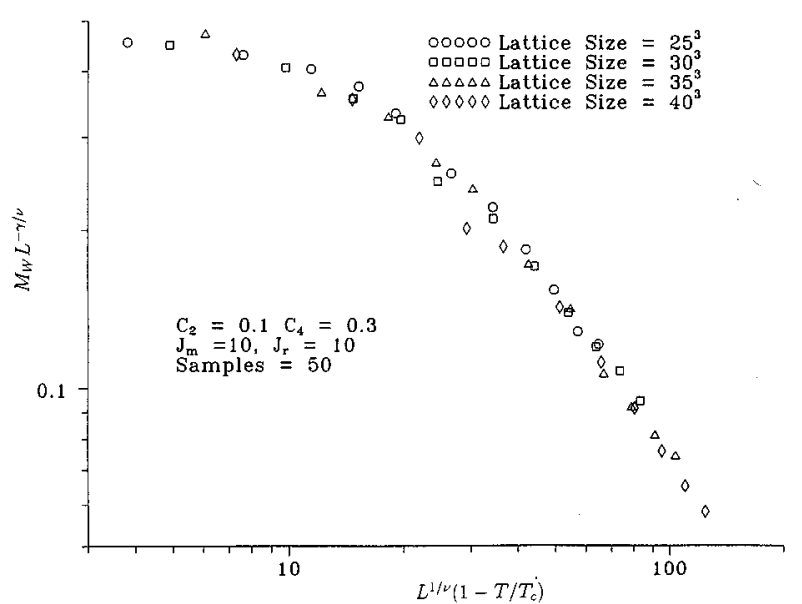

FIG. 6. Log-log plot of $M_{W} L^{-\gamma / \nu}$ vs $\left|1-T_{c} / T\right| L^{1 / \nu}$ with $J_{m}=10, J_{r}=10$. Assuming that $\gamma=1.42, \nu=0.73$.

The gel melting process can be thought of as the reverse of the gelation process. Ferry and Eldridge ${ }^{2}$ expressed the relationship between the concentration $C$ and the gelation (or melting) temperature $T_{g}$ by

$$
\ln C=\Delta H_{g} / R T_{g}+\text { const, }
$$

where $R$ is the universal gas constant and $\Delta H_{g}$ is the enthalpy of forming (or melting) the gel networks. This relation implies that the plot of $\ln C$ vs $1 / T_{g}$ has the slope $\Delta H_{g}$. Figure 9 shows the plot of $\ln C$ vs. $1 / T_{g}$ of the system described above.

In some systems, sol-to-gel and gel-to-sol transition temperatures are identical to each other, which implies that gelformation and gel-melting are equilibrium processes. ${ }^{4}$ In the system with crystalline junction points or helix junction points, the formation of gels is in two steps: fast conformational change is followed by slow intermolecular association. ${ }^{15,19}$ Gel-formation and gel-melting are nonequilibrium processes in those gels, which means that the

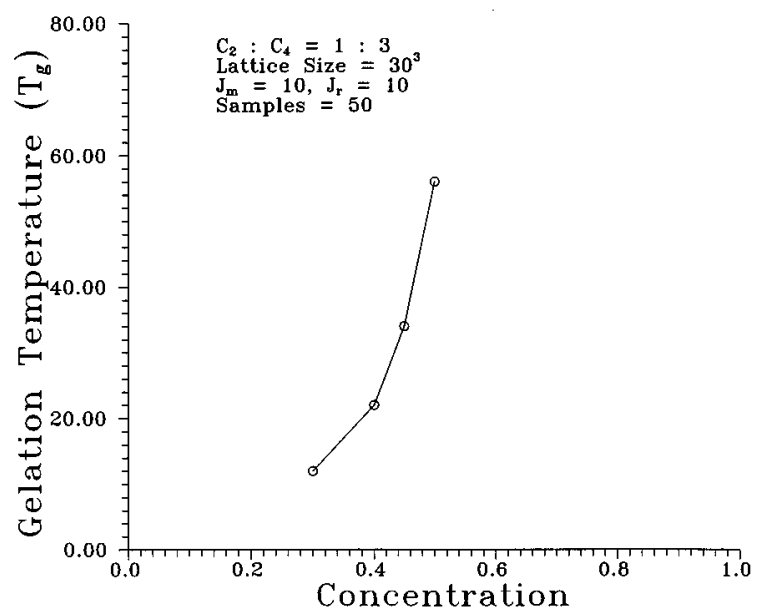

FIG. 8. Plot of gelation temperature $\left(T_{\text {gel }}\right)$ vs concentration of monomers $C=0.3,0.4,0.45, C_{2}: C_{4}=1: 3$ with $J_{m}=10, J_{r}=10$.

two temperatures are different. We can perform simulation with two levels of breakup energy, $J_{r 0}$ and $J_{r 1}$. We prepare a gel by starting from a mixture of unreacted monomers and let the polymerization proceed with a breakup energy parameter $J_{r 0}$ at a temperature $\left(T_{0}\right)$, which is below the gelmelting temperature. After the gel is formed, we quench the system to a finite temperature $T_{1}$ with a different breakup energy parameter $J_{r 1}$. The system will reach an equilibrium. If $T_{1}$ is above the gelation temperature, the gel network formed would melt due to thermal motions. Intuitively we know that if $J_{r 0}=J_{r 1}$, the system will have the same gelation temperature as the gel-melting temperature. Figure 10 shows the variations of the gel fraction versus the temperature with various breakup energy parameters $J_{r 1}$. The difference in the gelation temperature and the melting temperature is evident. We can see that if $J_{r 1}>J_{r 0}$, the melting temperature is greater than the gelation temperature.

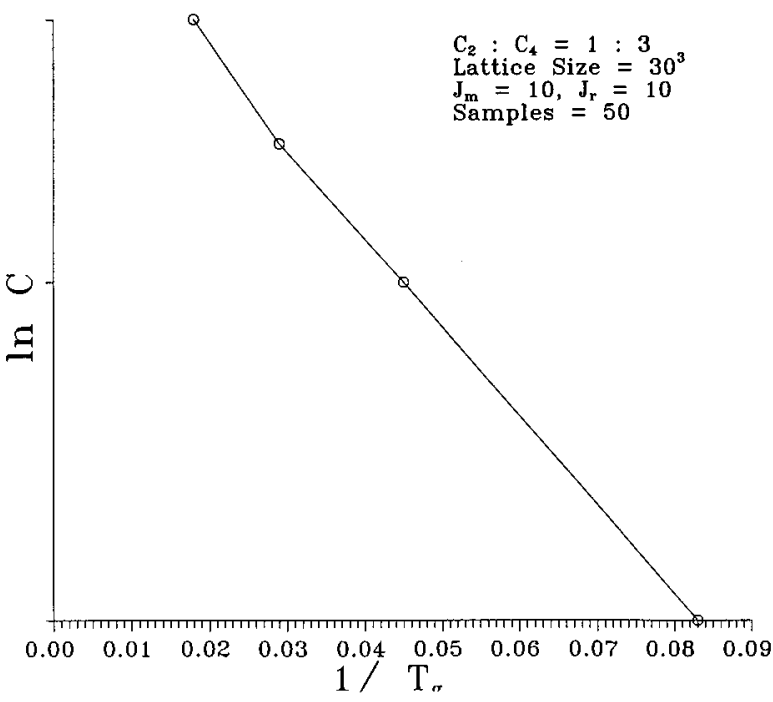

FIG. 9. Plot of $\ln C$ vs $1 / T_{\text {gel }}$ with $C_{2}: C_{4}=1: 3$ and $J_{m}=10, J_{r}=10$.
FIG. 7. Gel volume fraction vs temperature for various concentrations of monomers $C=0.3,0.4,0.45, C_{2}: C_{4}=1: 3$ with $J_{m}=10, J_{r}=10$.

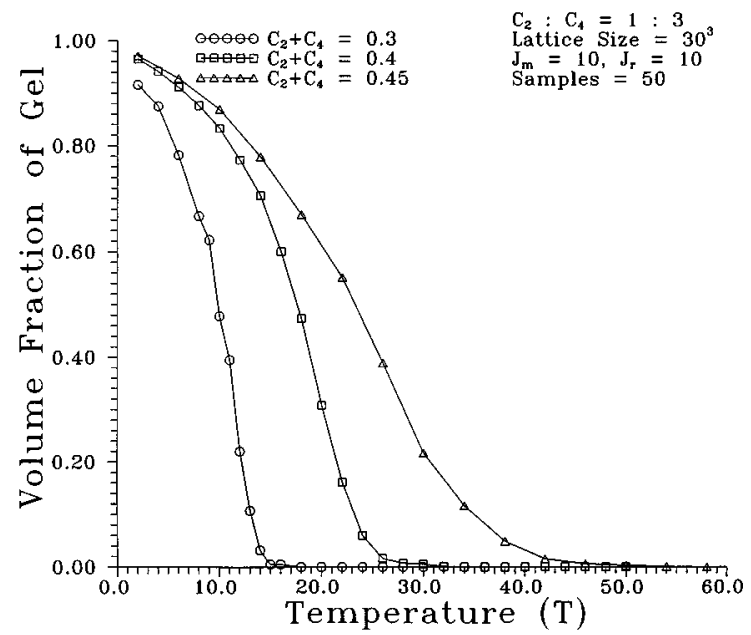

J. Chem. Phys., Vol. 105, No. 2, 8 July 1996 


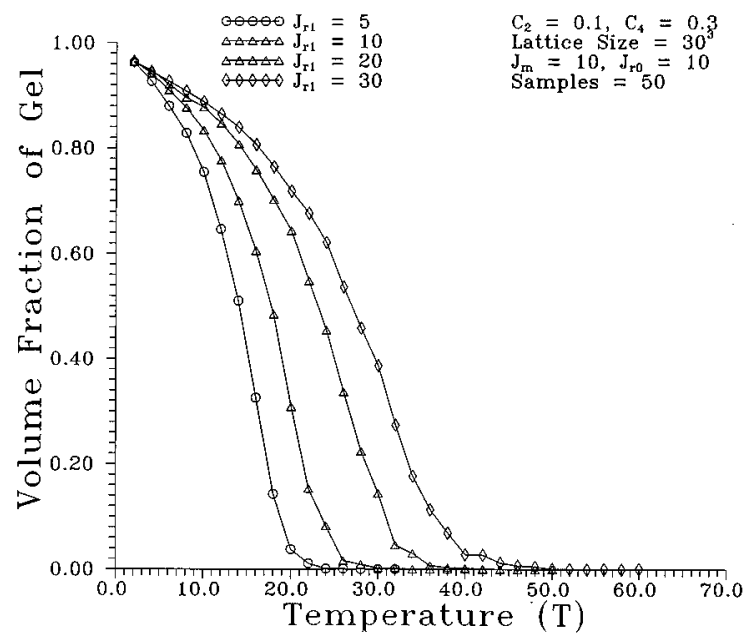

FIG. 10. Gel volume fraction vs temperature for various breakup energy parameter $J_{r 1}$ on the lattice $30^{3}$ with $C_{2}=0.1, C_{4}=0.3, J_{m}=10$ and $J_{r 0}=10$.

\section{B. Structural properties of a polymer-solvent mixture}

The thermodynamic properties of thermoreversible gels depend on the development of the gel structure in the gelation processes. In a gelation process, the system is cooled from a higher temperature, where the system is in a spatially homogeneous state. After a thermodynamically unstable state, the final equilibrium state is reached and a network is formed. During this process, the phase separation may also occur due to favorable interactions between the components of the system. The competition between phase separation and gelling process may lead to spatial inhomogeneity in the growth of a gel network. In this section we will study the kinetics of thermodynamically driven phase separation and connectivity-driven gelation process in a mixture of polymer, solvent, and empty sites.

We consider a lattice of size $40 \times 40 \times 40$ with a periodic boundary. The fraction $C_{4}$ of polymer units with a functionality $f=4$ is 0.3 and the length $n$ of the primary chains is 4 throughout this section. First we distribute these pre-bonded chains in the system by selecting $C_{4} \times L^{3} / n$ (the number of chains) lattice sites randomly from which the chains grow simultaneously (by random addition of monomers) until each chain grows 4 bonds. Then the fraction $C_{0}$ of the solvent particles is distributed in the lattice. The fraction of $1-C_{4}-C_{0}$ of lattice sites is empty. We investigate the effect of phase separation on the gelation process and final structures of gel networks with various bonding probabilities at various temperatures. The results for structure factors have been analyzed in order to study the kinetics of the phase separation and the gelation processes.

\section{Effects of cross-linking rate}

Without the gelation, the system in the one-phase region is thermodynamically stable. When a system of polymers and solvents is quenched from a thermodynamically stable state at a high temperature to a state at a lower temperature, it may evolve through relaxation patterns and may separate into two coexisting phases (polymer-rich and polymer-poor). This quenching process is similar to that in the gelation process discussed in the previous section. The cross-linking rate plays an important role in the gelation process with the coexistence of phase separation. Cross-linking may arrest the phase separation process.

In this simulation, we first quench the system from the infinite temperature, where the system is homogeneous in the sol phase, to a chosen temperature (i.e., $T=10.0$ ). The growth of microdomains, which are created by phase separation, is studied by monitoring the changes in the scattering pattern. Figure 11 shows the variation of the structure factor $S(q, t)$ as a function of $q^{\prime}=L q / 2 \pi$ with various bonding probabilities at different times (MC steps). We can see the effect of the bonding probability on the growth of microdomains from these figures. We know that the growth in the scattered intensity indicates a domain-forming process (the contrast between polymers and solvent). The microphase structures are expected for sufficiently small bonding probabilities $\left(p_{b}\right)$ which allow particles and small clusters to diffuse with higher probability. This is indeed demonstrated in Figures 11(a) and 11(b). The cross-linking rate has a strong effect on the mobility of components in the system. As the polymerization proceeds, clusters grow and diffuse more slowly. In the case of a higher bonding probability, the rate of growth is fast and the phase separation process slows down. This leads to pinning of the structure factor at the early stage [see Figure 11(c)]. We also observed that the position of the peak in the plot with a higher cross-linking rate moves towards larger $q$. This means that the interdomain spacings are different with different cross-linking rates.

To see the order of the microphase separation, we compare these data with the structure factors $S_{1}(q, t)$ which are sensitive to a possible solvent-vacancy phase separation. As the aggregation proceeds, the intensity grows, and the peak shifts towards smaller $q$ [Fig. 12(b)]. This is the typical pattern of a phase separation. We also evaluate the average contact number between polymer units and solvent particles, $n_{s p}$, i.e., the average number of the solvent particles adjacent to the polymer units in a configuration. This average can represent the degree of phase separation in the system. The larger this number, the bigger the microphase separated domain will be. Figure 13 shows the variation of $n_{s p}$ with time $t$. We see that, on increasing the bonding probability, the contact number $n_{s p}$ increases, i.e., the phase separation is suppressed due to cross-linking in the gelation processes. Thus, the rate of polymerizations and the rate of phase separation play a role in determining the structures of the gel networks.

\section{Temperature dependence}

It is expected that the resulting morphology of the gel depends on the temperature. For a system exhibiting USCT (Upper Solution Critical Temperature) behaviors, the chains are randomly dispersed in solution at a high temperature, where the thermal energy dominates over the interaction energy. As the temperature is reduced, the attractive intermolecular forces start to dominate, and the phase separation 

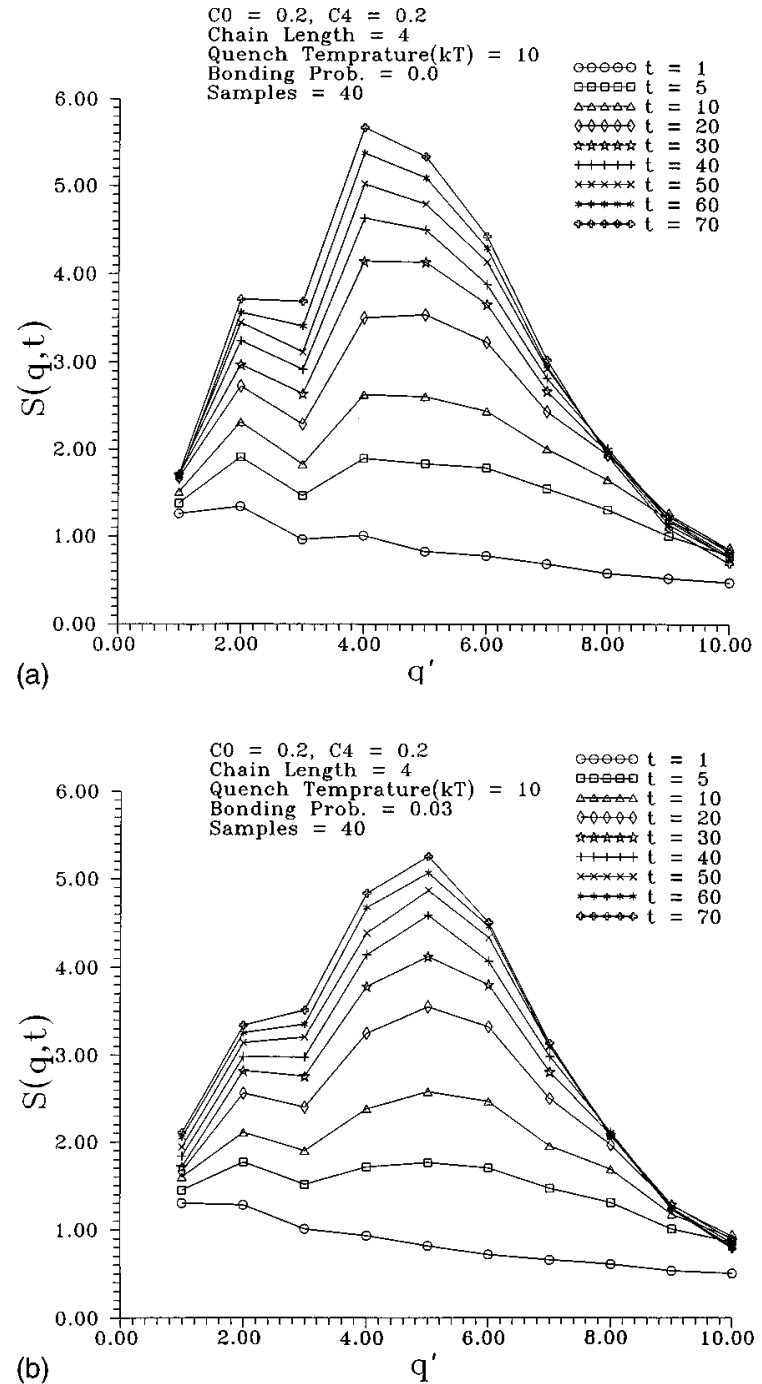

(b)

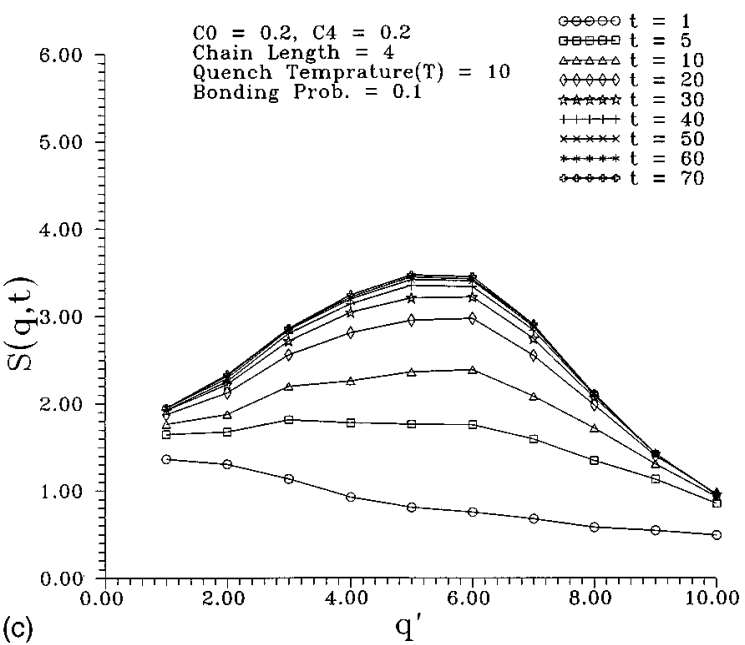

FIG. 11. The structure factors $S(q, t)$ plotted vs $q^{\prime}=q L / 2 \pi$ at various time (MC steps) for various crosslinking rate (bonding probability). (a) $p_{b}=0.0$; (b) $p_{b}=0.03$; (c) $p_{b}=0.1$ after quenched from infinite temperature to $T=10.0$.

may occur. In this section we will study the effect of temperature on the development of microdomains due to microphase separation in the gelation process. We choose two typical temperatures, $T=20.0$ and $T=30.0$, as the quenching
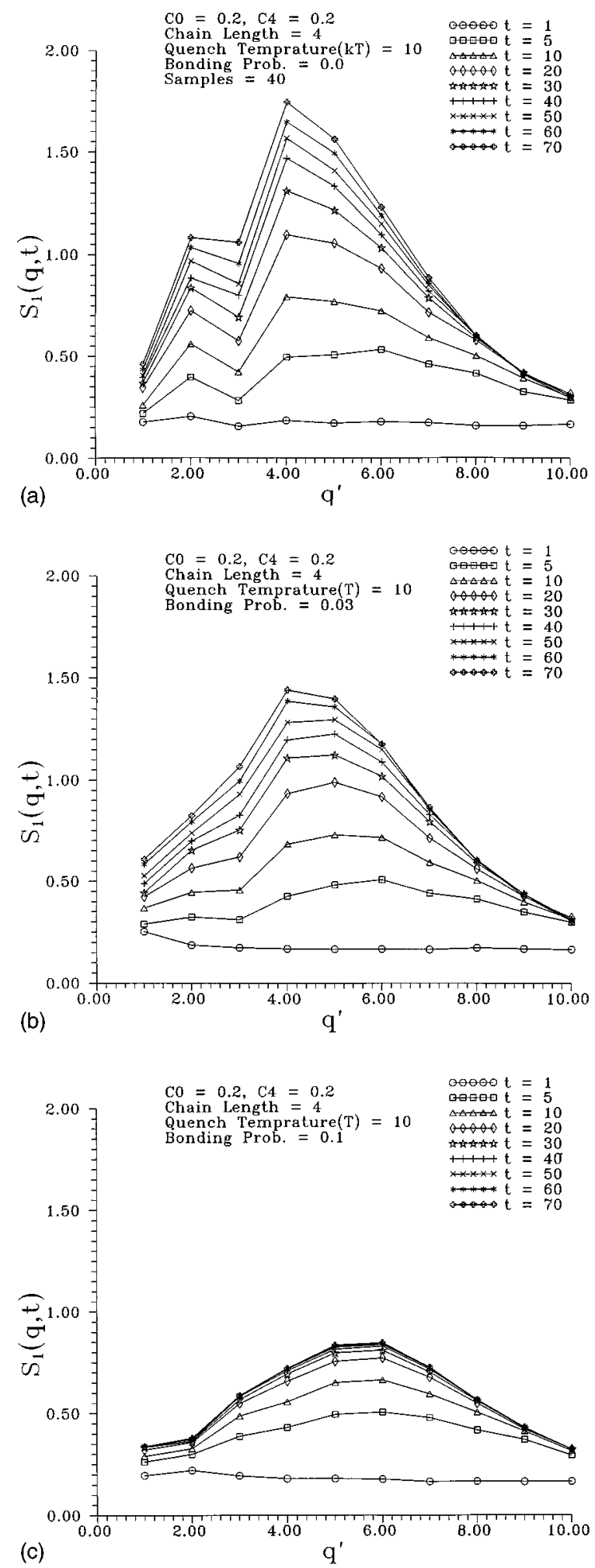

FIG. 12. The structure factors $S_{1}(q, t)$ for the solvent density fluctuation plotted vs $q$ at various time after the quench for various crosslinking rate. Parameters are the same as those in Figure 10.

temperatures and compare the profiles of the structure factor. Figures 14 and 15 are the plots of structure factor $S(q, t)$ as a function of $q^{\prime}=q L / 2 \pi$ at quench temperatures $T=20.0$ and $T=1.0$, respectively. For the system at high temperature 


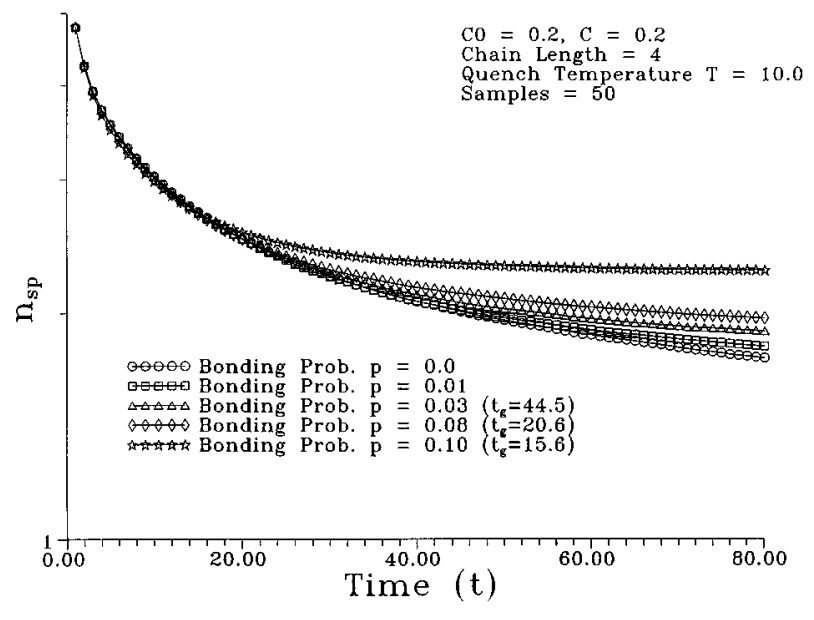

FIG. 13. Semi-log plot of number $n_{s p}(t)$ of contacts between polymer unit and solvent particles per particle vs time for various bonding probabilities with the quench temperature $T=10.0$.

$(T=20.0)$ (Figure 14), the phase separation effect is enhanced even though the association is not as strong at the lower temperature as at the higher temperature. This is because the bonds are formed and broken many times due to higher reversibility as polymer units and solvent particles are more mobile. So the rate of phase separation is faster than that of gelation and the scattering intensity is greater than that in the case of $T=1.0$, where the rate of growth is comparable and leads the pinning of the structure of the network. The position of peaks seems to shift to a small angle when the rate of the growth is low [see Figure 14(b)]. Notice that the profile of the structure factor in Figure 14 is broader in the regime of smaller angles compared with that in Figure 15. This suggests the disappearance of smaller domains and the gradual formation of bigger domains in later times.

From Figure 15 we note that the small angle part of the scattering intensity increases quite slowly. This means that an increase in the correlation length occurs because of the increased dominance of the interactions between polymer units at a lower temperature. The position of the peak in $S(q, t)$ does not seem to shift [Fig. 15(b)]. In the case of phase separation without gelation, as the domain grows, the scattering peak shifts to a lower angle and its intensity increases. But in many experiments, the intensity increases with time, while the peak remains stationary. This invariance of peak position can be a consequence of the impingement effect due to chemical reaction ${ }^{31}$ or the growth of some structures after the onset of gelation due to the lower reversibility of clusters and the larger extent of the network formation. ${ }^{28}$ In a real system, the domain may be smaller and ramified due to aggregations.

The effect of the temperature on the final morphology of gel networks can be seen clearly in Figs. 16(a) and 16(b), which are the plots of structure factor $S(q, t)$ as a function of $q^{\prime}=q L / 2 \pi$ at $\mathrm{t}=80 \mathrm{MCS}$ at various temperatures. The phase-separation process separates into two distinct parts. In part I [Fig. 16(a)], the intensity of the scattering increases as the temperature increases. In this regime, the reversibility of
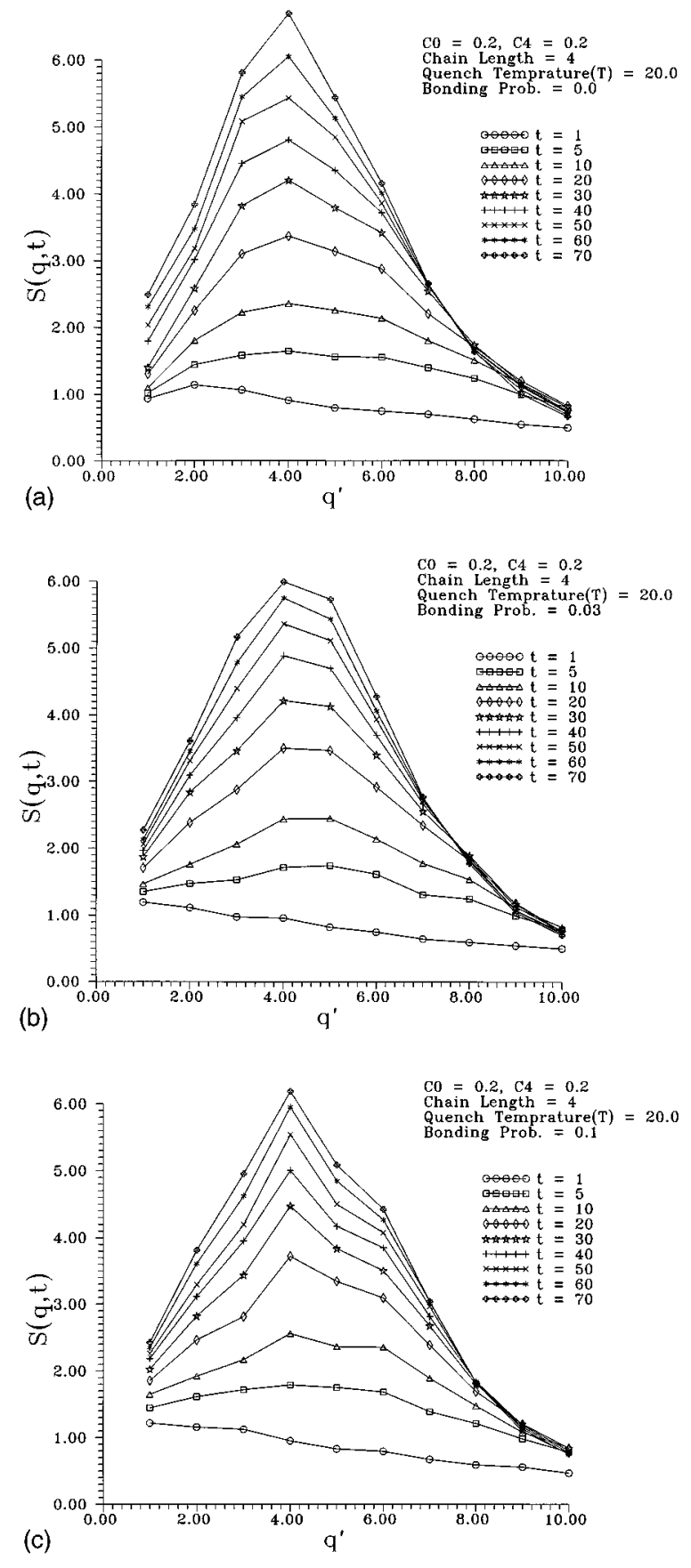

FIG. 14. The structure factors $S(q, t)$ plotted vs $q^{\prime}=q L / 2 \pi$ at various time (MC steps) for various crosslinking rate (bonding probability). (a) $p_{b}=0.0$; (b) $p_{b}=0.03$; (c) $p_{b}=0.1$ after quenched from the infinite temperature to $T=20.0$.

clusters is small. The rate of phase separation depends on the competition between the unfavorable interactions and the cross-linking reaction. At lower temperatures, the attractive interaction between the polymer units dominates. So, the rate of growth is faster in comparison with the phase-separation. The structure factor ceases to evolve at the early stage as the pinning of the structure by the cross-link reaction occurs. While at higher temperatures, the rate of the growth and the rate of phase-separation are comparable due to the weaker associations between polymer units. Therefore, the pinning 

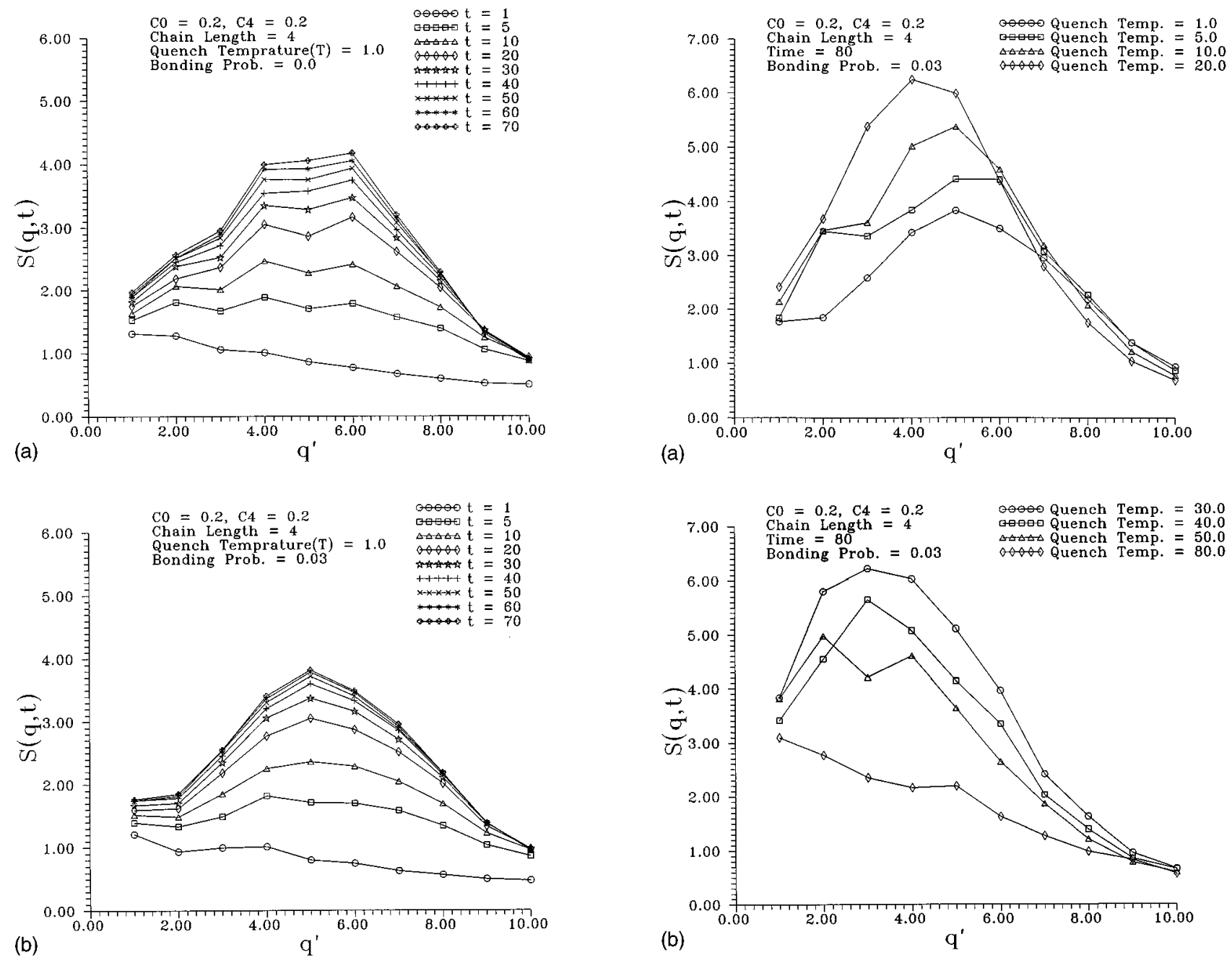

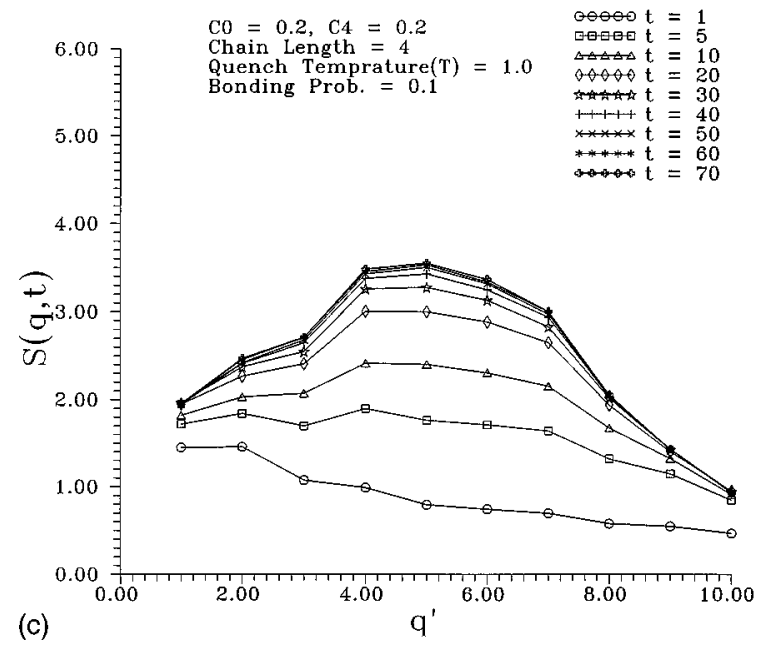

FIG. 15. The structure factors $S(q, t)$ plotted vs $q^{\prime}=q L / 2 \pi$ at various time (MC steps) for various crosslinking rate (bonding probability). (a) $p_{b}=0.0$; (b) $p_{b}=0.03$; (c) $p_{b}=0.1$ after quenched from the infinite temperature to $T=1.0$.

time is longer. The phase-separation at this regime has been studied by Feke et al. ${ }^{24}$ The structure of gel networks seems to develop from the co-continuous structure to fragmented structure as the quenching temperature changes.

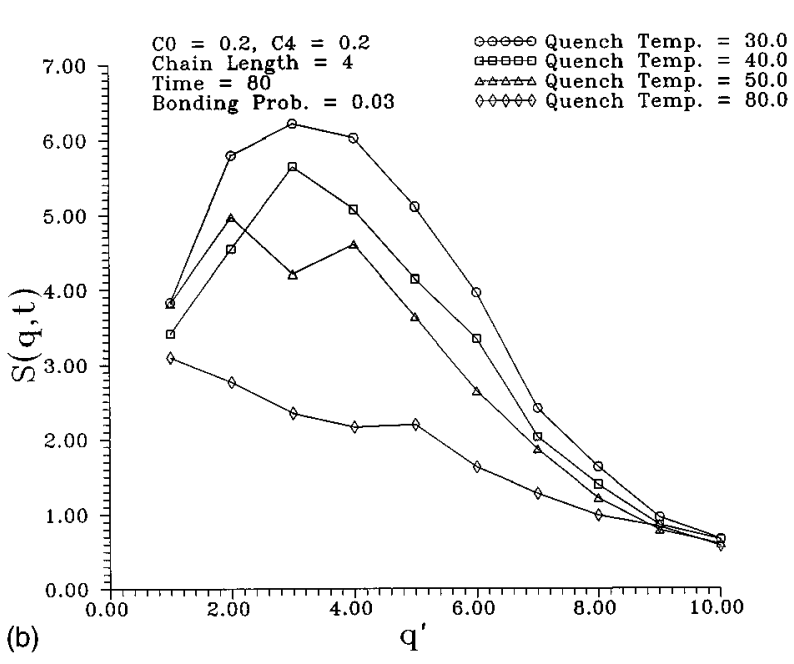

(b)

FIG. 16. The structure factors $S(q, t)$ plotted vs $q^{\prime}=q L / 2 \pi$ at time $t=80$ (MC steps) for various quench temperatures (a) $T=1.0,5.0,10.0$, and 20.0; (b) $T=30.0,40.0,50.0$, and 80.0.

In part II [Figure 16(b)], on the other hand, the reversibility dominates, which affects the phase-separation process. Notice that the gelation temperature is 22.0 for this system. In this part, no gel network is formed. We observe that the scattering intensity decreases at elevated temperatures. This is due to increased mobility of the polymer units and higher probability of breaking the clusters. The phase separation is suppressed and the system becomes more and more homogeneous as the temperature increases. We observed that the peak position of the structure factor shifts at different temperatures. This indicates that the structure of the system may be different even if the intensities have the same value at different temperatures. At the higher temperature regime, the position of the peaks moves towards smaller angles, i.e., the spread of the interdomain space is smaller because small clusters and chains diffuse between the domains without reaction due to the weaker interactions between the polymer units.

Since the process of gelation has an influence on the phase separation, it is impossible to get a "true" equilibrium 
phase diagram from the experiments, i.e., we cannot determine the spinodal curve in a phase diagram from the structure factor $S(q, t)$ due to the coexistence of the gelation and phase separation. But it is expected that the degree of inhomogeneities would be enhanced near the spinodal curve in the phase diagram. ${ }^{35}$ Therefore, one may obtain this temperature with larger lattices and finer temperature parameters.

\section{CONCLUSIONS}

A computer simulation model is developed to study the sol-gel transition in thermoreversible gels. In this model each particle (monomer, polymer, or microgel) is mobile. The clusters (microgel and gel) may also expand or contract due to bond fluctuation. The movement is correlated via energy parameters. Another feature of this model is the reversibility of the cluster, i.e., they can be formed and broken up due to their thermal motion. To relate the connectivity property of the gel network with the change in temperatures, we have used a simple bond fluctuation and breakup method in which cross-links can be broken due to the motion of polymer units. It provides a realistic description of the correlation between the chemical and the physical processes during solto-gel transition in polymeric systems.

We have studied the thermal transitions and structural properties of thermoreversible gels. The sol-gel transition of the thermoreversible gel is studied as a function of temperature. The critical exponents of the model seem to be the same as those in the percolation model. We have also studied the evolution of phase separation and gelation with various cross-linking rates and various quenching temperatures. From the analysis of data of static structure factors, we find that the final morphology of the macroscopic gel structure can be determined and that the domains of various shapes and sizes may emerge due to interplay between gelation and phase separation processes. The qualitative features of these structure factors are in agreement with the scattering experiments.

\section{ACKNOWLEDGMENTS}

This work is supported by a Cray Research grant and an NSF-EPSCoR research grant. The computer simulations are performed on Cray YMP and Cray J916 at the Mississippi Center for Supercomputing Research and work stations at the University of Southern Mississippi. A part of the manuscript was prepared at the National University of Singapore where R.B.P. was visiting the Computational Science Program; the warm hospitality is acknowledged by R.B.P.

${ }^{1}$ P. J. Flory, J. Am. Chem. Sci. 63, 3083,3091,3096(1941); P. J. Flory, Principles of Polymer Chemistry (Cornell University, New York, 1953).

${ }^{2}$ J. E. Eldridge and J. D. Ferry, J. Phys. Chem. 58, 992 (1954).

${ }^{3}$ P. G. de Gennes, Scaling Concepts in Polymer Physics (Cornell University, New York, 1979).
${ }^{4}$ H-M. Tan, A. Moet, A. Hiltner, and E. Baer, Macromolecules 16, 28 (1983).

${ }^{5}$ K. Kawanishi, M. Komatsu and T. Inoue, Polymer 28, 980 (1987).

${ }^{6}$ W. Burchard, R. Stadler, L. L. Freitas, M. Möller, J. Omeis and E. Mühleisen, in Biological and Synthetic Polymer Networks, edited by O. Kramer (Elsevier Applied Science, London, 1986).

${ }^{7}$ J. M. Guenet, Macromolecules 19, 1961 (1986).

${ }^{8} \mathrm{H}$. Berghmans and W. Stoks, in Integration of Fundamental Polymer Science and Technology, edited by L. A. Kleintjens and P. J. Lemstra (Elsevier Applied Science, New York, 1986).

${ }^{9}$ H. Berghmans, A. Donkers, L. Frenay, W. Stoks, F. E. De Schryver, P. Moldenaers, and J. Mewis, Polymer 28, 97 (1987).

${ }^{10} \mathrm{P}$. Russo, Reversible Polymer Gels and Related Systems, ACS Symposium Series 350 (American Chemical Society, Washington, DC, 1988).

${ }^{11}$ M. Adam and J. P. Aimè, J. Phys. II (Paris) 1, 1227 (1991).

${ }^{12}$ M. Klein, J.-M. Guenet, A. Brulet, and F. Boue, Polymer 32, 1943 (1991).

${ }^{13}$ J. C. Horton and A. M. Donald, Polymer 32, 2418 (1991).

${ }^{14}$ P. Shukla, Polymer 33, 365 (1992).

${ }^{15}$ M. Berghmans, S. Govaers, H. Berghmans, and F. C. De Schryver, Polym. Eng. Sci. 32, 1466 (1992)

${ }^{16}$ M. Okabe, K. Mitsui, H. Uranaka, M. Takahashi, and H. Matsuda, Polym. J, 24, 653 (1992).

${ }^{17}$ D. K. Rout, S. K. Pulapura, and R. A. Gross, Macromolecules 26, 6007 (1993).

${ }^{18}$ H. B. Bohidar and S. S. Jena, J. Chem. Phys. 98, 8970 (1993).

${ }^{19}$ M. Berghmans, S. Thijs, M. Cornette, H. Berghmans, and F. C. De Schryver, Macromolecules 27, 7669 (1994).

${ }^{20}$ N. Fazel, A. Brûlet, and J. M. Guenet, Macromolecules 27, 836 (1994).

${ }^{21}$ E. C. De Oliveira Lima and F. Galembeck, J. Colloid Interface Sci. 166, 309 (1994).

${ }^{22}$ C. Daniel, C. Dammer, and J.-M. Guenet, Polymer 35, 4243 (1994).

${ }^{23}$ S. Mal, P. Maiti, and A. K. Nandi, Macromolecules 28, 2371 (1995).

${ }^{24}$ G. T. Feke and W. Prins, Macromolecules 7, 527 (1974).

${ }^{25}$ S. P. Nunes and B. A. Wolf, Macromolecules 20, 1952 (1987).

${ }^{26}$ I. M. Low and R. McPherson, J. Mater. Sci. 23, 4141 (1988).

${ }^{27}$ M. He, Y. Liu, Y. Feng, M. Jiang and C. C. Han, Macromolecules 24, 464 (1991).

${ }^{28}$ R. Bansil, J. Lal and B. L. Carvalho, Polymer 33, 2961 (1992).

${ }^{29}$ Y. Cohen, O. Ramon, I, J. Kopelman, and S. Mizrahi, J. Polym. Sci. B 30, 1055 (1992)

${ }^{30}$ J. Y. Kim, C. H. Cho, P. Palffy-Muhoray, M. Mustafa, and T. Kyu, Phys. Rev. Lett. 71, 2232 (1993).

${ }^{31}$ E. K. Hobbie, B. J. Bauer, and C. C. Han, Phys. Rev. Lett. 72, 1839 (1994).

${ }^{32}$ K. F. Silveira, I. V. P. Yoshida, and S. P. Nunes, Polymer 36, 1425 (1995).

${ }^{33}$ A. Prasad, H. Marand, and L. Mandelkern, J. Polym. Sci. B 32, 1819 (1993).

${ }^{34}$ T. Kyu, J. C. Yang, S. Z. D. Cheng, S. L. C. Hsu, and F. W. Harris, Macromoleules 27, 1861 (1994).

${ }^{35}$ E. S. Matsuo, M. Orkisz, S-T. Sun, Y. Li, and T. Tanaka, Macromolecules 27, 6791 (1994)

${ }^{36}$ S. M. Kobayashi, T. Yoshioka, T. Kozasa, K. Tashiro, J.-I. Suzuki, S. Funahashi, and Y. Izumi, Macromolecules 27, 1349 (1994).

${ }^{37}$ D. Asnaghi, M. Giglio, A. Bossi, and P. G. Righetti, J. Chem. Phys. 102, 9736 (1995).

${ }^{38}$ F. Tanaka and A. Matsuyama, Phys. Rev. Lett. 62, 2759 (1989); F. Tanaka, Macromolecules 23, 3784,3790 (1990); F. Tanaka and M. Ishida, Physica A 204, 660 (1994); F. Tanaka and W. H. Stockmayer, Macromolecules 27, 3943 (1994).

${ }^{39}$ A. Coniglio, H. E. Stanley, and W. Klein, Phys. Rev. Lett. 42, 518 (1979); Phys. Rev. B 25, 6805 (1982).

${ }^{40}$ S. C. Glotzer, M. F. Gyure, F. Sciortino, A. Coniglio, and H. E. Stanley, Phys. Rev. Lett. 70, 3275 (1993); S. C. Glotzer, M. F. Gyure, F. Sciortino, A. Coniglio, and H. E. Stanley, Phys. Rev. E 49, 247 (1994).

${ }^{41}$ F. Sciortino, R. Bansil, and H. E. Stanley, Phys. Rev. E 47, 4615 (1993).

${ }^{42}$ D. Stauffer, A. Coniglio, and M. Adam, Adv. Polym. Sci. 44, 103 (1982).

${ }^{43}$ S. C. Glotzer, R. Bansil, P. D. Gallagher, M. F. Gyure, F. Sciortino, and H. E. Stanley, Physica A 201, 482 (1993).

${ }^{44}$ W. H. Jo and M. B. Ko, Macromolecules 27, 7815 (1994).

${ }^{45}$ M. Schulz and H. L. Frisch, J. Chem. Phys. 101, 10008 (1994). 
${ }^{46}$ Y. Liu and R. B. Pandey, J. Phys. II (Paris) 4, 865 (1994)

${ }^{47}$ H. M. J. Boots and R. B. Pandey, Polym. Bull. 11, 415 (1984).

${ }^{48}$ H. M. J. Boots, in Biological and Synthetic Polymer Networks, edited by O. Kramer (Elsevire, London, 1988), p. 267; H. M. J. Boots, Physica A 147, 90 (1987).

${ }^{49} \mathrm{~K}$. Binder, in Computational Modeling of Polymers, edited by J. Bicerano (Dekker, City, 1992); K. Binder, Adv. Polym. Sci. 112, 181 (1994).

${ }^{50}$ A. Sariban and K. Binder, Macromolecules 24, 578 (1991).
${ }^{51}$ A. L. Rodriguez, J. J. Freire, and A. Horta, J. Phys. Chem. 96, 3954 (1992).

${ }^{52}$ L. A. Molina and J. J. Freire, Macromolecules 28, 2705 (1995).

${ }^{53}$ D. Stauffer and A. Aharony, Introduction to Percolation Theory (Taylor \& Francis, London, 1994).

${ }^{54}$ K. Binder and D. W. Heermann, Monte Carlo Simulation in Statistical Physics (Springer, Berlin, 1988). 
The Journal of Chemical Physics is copyrighted by the American Institute of Physics (AIP). Redistribution of journal material is subject to the AIP online journal license and/or AIP copyright. For more information, see http:/ojps.aip.org/jcpo/jcpcr/jsp Copyright of Journal of Chemical Physics is the property of American Institute of Physics and its content may not be copied or emailed to multiple sites or posted to a listserv without the copyright holder's express written permission. However, users may print, download, or email articles for individual use. 
The Journal of Chemical Physics is copyrighted by the American Institute of Physics (AIP). Redistribution of journal material is subject to the AIP online journal license and/or AIP copyright. For more information, see http://ojps.aip.org/jcpo/jcper/jsp 